\section{NextNet Wireless}

NextNet, the industry's most widely deployed provider of non-line-of-sight (NLOS) plug-and-play broadband wireless access systems has an immediate opening for a Software Engineer. This position will be responsible for the design, development and support of RISC based embedded software for interfacing to proprietary signal processing devices and microprocessor support chips. You will be part of a team that will be extending the features and capabilities of the existing wireless data system. Resumes to: hr@nextnetwireless.com.

\section{The Oak Ridge National}

\section{Laboratory}

Director of the Center for

Computational Sciences (CCS)

The Oak Ridge National Laboratory (ORNL) in East Tennessee seeks an outstanding scientist with exceptional leadership experience and skills to serve as the Director of the Center for Computational Sciences (CCS) and the recently announced National Leadership-Class Computing Facility (NLCF), in accordance with the recently announced U.S. Department of Energy's Office of Science, Advanced Scientific Computing Research initiatives.

The CCS is the premier organization at ORNL for applied computational sciences, high-performance research systems and intelligent and emerging computational systems. The position reports to the Associate Laboratory Director for Computing and Computational Sciences http://www.ornl.gov info/news/cco/ccsfact.pdf).

This high-profile position will require managing a base of activities (basic research, applied research, systems, services, engineering, technical development, assessment, etc.) and multidisci- plinary research project areas (organized as institutes) that are highly complex and represent a significant portion of the Center's work. The CCS Institutes are an outreach activity, providing an intellectual home and computational infrastructure for community building. The Institutes share common goals for promoting scientific discovery and application. The ideal candidate will have an advanced technical degree with extensive training and experience in the computational sciences. The candidate must also have a proven ability to plan, organize, lead and manage activities of a major organizational unit within a major R\&D facility, including a working knowledge of policies and guidelines that relate to administration and management of complex R\&D organizations. Candidates must, of course, possess excellent communications skills and a strong desire to work in a team environment.

Inquiries and expressions of interest may be directed to: Staffing, Computing and Computational Sciences Directorate, Oak Ridge National Laboratory, P.O. Box 2008, Oak Ridge, TN 37831-6163; email: CCSD_Staffing@ornl.gov. Please include the number of the position for which you are applying in the subject line of your email.

Alternatively, interested candidates may contact our search consultant in full confidence: P.J. O'Hare, Executive Search Consultant, 2956 Missy Drive NE, Marietta, GA 30062-4329; phone: (404) 642-9105; email: pjosearch@min dspring.com

More information about the Oak Ridge National Laboratory is available at http://www.ornl.gov

ORNL, a multiprogram research facility managed by UT-Battelle, LLC, for the U.S. Department of Energy, is an equal opportunity employer committed to building and maintaining a diverse work force.

\section{The Oak Ridge National}

\section{Laboratory}

\section{Visualization Task Leader}

The Computer Science and Mathematics Division of Oak Ridge National Laboratory (ORNL) seeks a Visualization Task Leader to perform research in advanced visualization and to manage the production PowerWall and reconfigurable CAVE Automatic Virtual Environment facilities.

The successful candidate will be responsible for integrating interdisciplinary teams of computer scientists, applications scientists, mathematicians and engineers in high-end visualization applications; immersive virtual reality; high-end conventionally driven systems; pc-based, cluster-driven systems; collaborative desktop-to-CAVE environments; advanced rendering techniques; and advanced interactive visualization.

The position requires a $\mathrm{PhD}$ in computer science or one of the computational sciences (or a closely related discipline) and extensive management and research experience with visualization user facilities. Candidates must have a proven record of research accomplishments, excellent communications, interpersonal and organizational skills and a strong desire to work in a team environment. Experience managing client-vendor-contractor relationships and contracts is required, as is supervisory experience and strong personal motivation. Project management experience is preferred. Hands-on parallel computing and visualization creation experience is desirable.

Inquiries and expressions of interest may be directed to: Staffing, Computing and Computational Sciences Director- 
ate, Oak Ridge National Laboratory, P.O. Box 2008, Oak Ridge, TN 37831-6163; email: CCSD_Staffing@ornl.gov. Please include the number of the position for which you are applying in the subject line of your email.

Alternatively, interested candidates may contact our search consultant in full confidence: P.J. O'Hare, Executive Search Consultant, 2956 Missy Drive NE, Marietta, GA 30062-4329; phone: (404) 642-9105; email: pjosearch@min dspring.com

More information about the Oak Ridge National Laboratory, the Computer Science and Mathematics Division, and Visualization is available at: http://www.ornl.gor http:/ www.csm.ornl.gov/ or http://www.csm. ornl.gov/viz/index.htm

ORNL, a multiprogram research facility managed by UT-Battelle, LLC, for the U.S. Department of Energy, is an equal opportunity employer committed to building and maintaining a diverse work force.

\section{The Oak Ridge National \\ Laboratory \\ Project Manager}

The basic function of this position is to investigate the architecture of and software systems for high-end computer systems. Areas of interest include microprocessor and interconnect technology, computer systems performance evaluation and simulation, performance modeling and prediction, tools for performance analysis, programming languages, messaging software, emerging programming models, workload characterization, fault-tolerance, operating systems for HPC, and use of advanced statistical methods and machine learning for performance analysis.

Major duties and responsibilities: The successful candidate will analyze architecture and application performance of on-site high performance computers to be located in the DOE's National Leader-

ship Computing Facility (NLCF), create and deploy prototype software, publish results in journals and at conferences, and collaborate with external researchers. Research activities include, but are not limited to:

- Evaluation of high-performance software systems and testing the effectiveness of supercomputer architectures in solving complex problems in biology, astrophysics and other scientific disciplines.

- Studying the performance of highperformance computer systems (both vector processing and massively parallel processing) under various workload characteristics through measurement, modeling and simulation.

- Publishing papers in high-quality refereed conferences and journals.

- Actively collaborating with partner government laboratories, academia, and industry applications developers. Education, experience, skills: The position requires an advanced degree $(\mathrm{PhD}$ preferred) in computer science or one of the computational sciences, with five or more years of recent leading-edge research experience with experimental high-performance computing software and parallel computer architectures. Requires demonstrated experience in software development on parallel computers using message passing, OpenMP,

\section{Advertising in Career Opportunities}

How to Submit a Classified

Line Ad: Send an e-mail to acmadvertising@acm.org. Please include text, and indicate the issue/or issues where the ad will appear, and a contact name and number.

Estimates: An insertion order will then be e-mailed back to you. The ad will by typeset according to CACM guidelines. No proofs can be sent. Classified line ads are not commissionable.

Rates: $\$ 240.00$ for six lines of text, 40 characters per line. $\$ 80.00$ for each additional three lines. The minimum is six lines. and Fortran 90, C, or C++, along with expertise in architectures, operating systems, compilers, runtime systems and programming tools.

Candidates must have a proven record of research accomplishments and a strong desire to participate in research efforts in a team environment. Demonstrated experience communicating clearly and concisely both orally and in writing is also needed.

The chosen candidate will be required to obtain an $\mathrm{L}$ clearance.

Inquiries and expressions of interest may be directed to: Staffing, Computing and Computational Sciences Directorate, Oak Ridge National Laboratory, P.O. Box 2008, Oak Ridge, TN 37831-6163; email: CCSD_Staffing@ornl.gov. Please include the number of the position for which you are applying in the subject line of your email.

Alternatively, interested candidates may contact our search consultant in full confidence: P.J. O'Hare, Executive Search Consultant, 2956 Missy Drive NE, Marietta, GA 30062-4329; phone: (404) 642-9105; email: pjosearch@min dspring.com

More information about the Oak Ridge National Laboratory is available at http://computing.ornl.gov, http:// www.ccs.ornl.gov/nlcf and http:/ www.ornl.gov
Deadlines: The closing date is on the 21 st of the month, five weeks prior to the publication date of the issue (which is the first of every month).

Career Opportunities Online: Classified and recruitment display ads receive a free duplicate listing on our website at http://www.acm.org/ $\mathrm{cacm} /$ careeropps/. Ads are listed for a period of six weeks.

For More Information Contact: William Kooney, Account Manager, at 212-626-0687 or kooney@hq.acm.org 
ORNL, a multiprogram research facility managed by UT-Battelle, LLC, for the U.S. Department of Energy, is an equal opportunity employer committed to building and maintaining a diverse work force.

\section{The Oak Ridge National}

\section{Laboratory}

\section{Group Leader for Scientific}

\section{Applications Support}

The Group Leader for Scientific Applications Support in the DOE Center for Computational Sciences is responsible for managing and conducting leading-edge research and application support for the users of high-performance computing (HPC) systems and for performing research on HPC tools and algorithms. Support will cover a wide range of HPC applications, such as climate modeling, astrophysics, materials science, chemistry, and database searching. The major duties and responsibilities of the position include, but are not limited to, the following:

- Develop and lead a successful program in scientific applications research (quality of science, staffing and budget oversight).

- Interact with the appropriate program offices and other agencies to develop and maintain program funding for basic and applied research.

- Perform and publish high quality research.

- Collaborate with application scientists in the Laboratory on advancing the scientific mission of the Laboratory.

- Take responsibility for personnel management, including but not limited to, creation of personnel performance appraisals and employee development plans.

- Represent group-level work to review committees and outside non-government bodies as appropriate.

- Program development and development of new research directions as a basis of continued funding and the overall health of the Group.

- Provide leadership in all areas of ES\&H and full implementation of integrated safety management in the Group.

- Ensure staff compliance with Laboratory policies, standards and procedures and other regulations.

- Select and retain highly qualified staff, develop capabilities of staff, and ensure that staff is competent, trained and qualified for assigned work.

- Provide effective and meaningful performance feedback to individual staff members.

- Ensure that staff are effectively utilized, rewarded and motivated.

- Support the professional development of staff consistent with Laboratory and individual development plans.

- Prepare plans and budgets, and manage assigned resources (people, equipment, facilities).

Experience and Education Requirements: An advanced degree in computer science (or an equivalent combination of education and experience). Additionally, the position requires five years of directly related research experience with a substantial publications record in HPC research and applications. Requires a demonstrated ability to develop and maintain research programs and substantial programmatic funding. Personnel management skills are essential, along with the ability to communicate effectively verbally and in writing. Knowledge of government laboratory business practices is a plus, specifically within the Department of Energy or related federal departments. Candidates must have a proven capability to work effectively in a team environment.

Inquiries and expressions of interest may be directed to: Staffing, Computing and Computational Sciences Directorate, Oak Ridge National Laboratory, P.O. Box 2008, Oak Ridge, TN 37831-6163; email: CCSD_Staffing@ornl.gov. Please include the number of the position for which you are applying in the subject line of your email.

Alternatively, interested candidates may contact our search consultant in full confidence: P.J. O'Hare, Executive Search Consultant, 2956 Missy Drive NE, Marietta, GA 30062-4329; phone: (404) 642-9105; email: pjosearch@min dspring.com

More information about the Oak Ridge National Laboratory is available at http://computing.ornl.gov and http: //www.ornl.gov

ORNL, a multiprogram research facility managed by UT-Battelle, LLC, for the U.S. Department of Energy, is an equal opportunity employer committed to building and maintaining a diverse work force.

\section{The Oak Ridge National Laboratory} Research Staff Member

Basic Function of Position: Research Staff Member to provide technical support skills and program development experience in the Systems Engineering and Technology Group that supports development of fundamental and applied research across ORNL in advanced prototype database concepts. Position will involve developing, enhancing and managing a prototype database in support of Homeland Security. The staff member will work as part of an ORNL multidisciplinary team consisting of hardware, software, database and programming specialists.

Major Duties and Responsibilities: There are multiple requirements for this position. The main responsibility will be to manage, maintain and support database development in a prototype system currently under design. This will include design of conversion codes from any number of formats to SQL for database population purposes. Individual may be required to develop software applications for analytical staff in a number of languages on short notice, help design taxonomies and entity relationships, 
post information to web portals and verify validity of data population processes. Experience Education and Skills Requirements: Position requires a minimum of a BS in Computational Sciences, Computer Programming or System Engineering with at least three years (preferably five) of experience in a relevant area of database or software development for prototype systems. Candidates should be familiar with both Microsoft and Linux operating systems. Requires experience in relational database design and implementation using a relational database management system (RDBMS) such as Microsoft SQL Server, Oracle 9i or 10g. Requires some experience troubleshooting and resolving hardware and/or network problems. Expertise with Veritas or Bru software is a plus.

The candidate should be highly motivated with good communications skills and a desire to work with multidisciplinary teams as well as initiate good research concepts and protocols. Strong problem solving ability under novel and/or less structured environments is highly desirable. Some travel may be required.

The chosen candidate will be required to obtain a $\mathrm{Q}$ clearance.

Inquiries and expressions of interest may be directed to: Staffing, Computing and Computational Sciences Directorate, Oak Ridge National Laboratory, P.O. Box 2008, Oak Ridge, TN 37831-6163; email: CCSD_Staffing@ornl.gov. Please include the number of the position for which you are applying in the subject line of your email.

Alternatively, interested candidates may contact our search consultant in full confidence: P.J. O'Hare, Executive Search Consultant, 2956 Missy Drive NE, Marietta, GA 30062-4329; phone: (404) 642-9105; email: pjosearch@min dspring.com

More information about the Oak Ridge National Laboratory is available at http://www.ornl.gov
ORNL, a multiprogram research facility managed by UT-Battelle, LLC, for the U.S. Department of Energy, is an equal opportunity employer committed to building and maintaining a diverse work force.

\section{The Oak Ridge National \\ Laboratory}

\section{Computer Scientist, Large Scale}

\section{Simulations}

The Computer Science and Mathematics Division at the Oak Ridge National Laboratory (ORNL) is the premier division for research in high-performance computing, computer science and applied mathematics. Basic and applied research programs focus on ultra-scale computation and the scientific applications that require it.

The successful candidate will join an interdisciplinary team of computer scientists, mathematicians and engineers working at the National Leadership Computing Facility (NLCF) on projects of national interest including fusion simulation, nanotechnology modeling, genomics, and computational chemistry.

Position requires a $\mathrm{PhD}$ in computational science or a related discipline with 3-5 years of research experience, or an equivalent combination of education and experience. Candidates must have a proven record of research accomplishments, including a strong record of scientific publications. Candidates should have excellent communications and a strong desire to work in a team environment. Project management experience is preferred. Some travel may be required.

Inquiries and expressions of interest may be directed to: Staffing, Computing and Computational Sciences Directorate, Oak Ridge National Laboratory, P.O. Box 2008, Oak Ridge, TN 37831-6163; email: CCSD_Staffing@ornl.gov. Please include the number of the position for which you are applying in the subject line of your email.

Alternatively, interested candidates may contact our search consultant in full confidence: P.J. O'Hare, Executive Search Consultant, 2956 Missy Drive NE, Marietta, GA 30062-4329; phone: (404) 642-9105; email: pjosearch@min dspring.com

More information about the Oak Ridge National Laboratory and the Computer Science and Mathematics Division is available at: www.ornl.gov

ORNL, a multiprogram research facility managed by UT-Battelle, LLC, for the U.S. Department of Energy, is an equal opportunity employer committed to building and maintaining a diverse work force.

\section{ACM Policy on}

\section{Nondiscriminatory Advertising}

ACM accepts recruitment advertising under the basic premise the advertising employer does not discriminate on the basis of age, color, race, religion, gender, sexual preference, or national origin.

ACM recognizes, however, that laws on such matters vary from country to country and contain exceptions, inconsistencies, or contradictions. This is true of laws in the United States of America as it is of other countries.

Thus ACM policy requires each advertising employer to state explicitly in the advertisement any employer restrictions that may apply with respect to age, color, race, religion, gender, sexual preference, or national origin. (Observance of the legal retirement age in the employer's country is not considered discriminatory under this policy.) ACM also reserves the right to unilaterally reject any advertising.

ACM provides notices of positions available as a service to the entire membership. ACM recognizes that from time to time there may be some recruitment advertising that may be applicable to a small subset of the membership, and that this advertising may be inherently discriminatory. ACM does not necessarily endorse this advertising, but recognizes the membership has a right to be informed of such career opportunities. 